\title{
INFERENCIAS PALEOAMBIENTALES A PARTIR DEL ANÁLISIS POLÍNICO DE SITIOS ARQUEOLÓGICOS DEL ÁREA DEL LAGO SAN MARTÍN (SANTA CRUZ, ARGENTINA)
}

\author{
FLORENCIA P. BAMONTE*, M. VIRGINIA MANCINI*, JUAN \\ BAUTISTA BELARDI** \& SILVANA ESPINOSA **
}

\begin{abstract}
RESUMEN
En la provincia de Santa Cruz fueron realizados diversos estudios paleoambientales relacionados con gradientes ambientales a partir de distintos tipos de depósitos. Los registros fósiles de cuevas y aleros (depósitos importantes para la reconstrucción de los ambientes del pasado en zonas extra-andinas) son el resultado de interacciones ambientales, climáticas y culturales que deben ser tenidas en cuenta para realizar reconstrucciones de la vegetación del pasado e inferir condiciones ambientales. Para este trabajo se analizaron dos secuencias polínicas fósiles provenientes de los sitios arqueológicos Bloque 1-Oquedad y Cueva del Paisano Desconocido en el área del lago San Martín (49 ${ }^{\circ} \mathrm{S} ; 72^{\circ} \mathrm{O}$ ). El objetivo fue integrar esta información a un marco paleoambiental generado a partir del análisis polínico y de carbón de Mallín La Tercera y a la información precedente sobre las ocupaciones humanas para el área. A partir del sitio Bloque 1-Oquedad, fue posible reconstruir las variaciones de la vegetación a escala local. En cambio, el análisis de la secuencia de la Cueva del Paisano Desconocido, brindó información similar a la secuencia Mallín La Tercera. Si bien no se observan cambios significativos en las comunidades de vegetación por parte de los grupos cazadores-recolectores para el área de estudio, los principales signos de impacto antrópico se registran para el último siglo (a partir de Mallín La Tercera) y son coincidentes con el asentamiento europeo.
\end{abstract}

PALABRAS CLAVE: lago San Martín, inferencias paleoambientales, análisis polínico de secuencias fósiles, ocupación humana.

*Laboratorio de Paleoecología y Palinología. Departamento de Biología. Facultad de Ciencias Exactas y Naturales. Universidad Nacional de Mar del Plata. IIMyC-CONICET. Mar del Plata, Provincia de Buenos Aires, Argentina. bamonte@mdp.edu.ar. mumancin@mdp.edu.ar.

**CONICET. Universidad Nacional de la Patagonia Austral. Unidad Académica Río Gallegos. Santa Cruz, Argentina.

silespinosa@gmail.com; juanbautistabelardi@gmail.com. 


\title{
PALAEOENVIRONMENTAL INFERENCES FROM ARCHAEOLOGICAL SITE POLLEN ANALYSIS AT LAKE SAN MARTÍN AREA (SANTA CRUZ, ARGENTINA)
}

\begin{abstract}
Several palaeoenvironmental studies related to environmental gradients from different types of deposits were made in Santa Cruz Province. Fossil records from caves and shelters (important deposits for the past environmental reconstructions in extra-Andean zones) are the result of environmental, climatic and cultural interactions. They must be taken into account to make past vegetation reconstruction and inferred environmental conditions. In this study were analyzed two pollen fossil sequences from the archaeological sites Bloque 1-Oquedad and Cueva del Paisano Desconocido from lake San Martin area (49 $\left.{ }^{\circ} \mathrm{S} ; 72^{\circ} \mathrm{W}\right)$. The aim was to integrate this information to a palaeoenvironmental context generated from pollen and charcoal analysis from Mallin La Tercera and prior information about human occupations in the area. From Bloque 1-Oquedad, was possible reconstruct local vegetation variations. On the other hand, the analysis of Cueva del Paisano Desconocido sequence, gave similar information to the mallin La Tercera sequence. Although there are not significant changes in vegetation communities by hunter-gatherers for the study area, the main signs of human impact are recorded during the last century (from Mallin La Tercera) and this is coincident with European settlement.
\end{abstract}

KEY WORDS: lake San Martin, palaeoenvironmental inferences, pollen analysis from fossil sequences, human occupation.

\section{INTRODUCCIÓN}

El sur de Sudamérica es un área importante para las investigaciones de las variaciones actuales y pasadas del sistema climático en el Hemisferio Sur. La Cordillera de los Andes funciona como una notable barrera topográfica para el paso de las masas de aire húmedo provenientes del Pacífico, generando una disminución exponencial de la precipitación hacia el este (Garreaud et al. 2009; Paruelo et al. 1998) que se ve reflejado en la distribución de la vegetación. Las características climáticas y geomorfológicas del sur de Patagonia posibilitan el estudio de los mecanismos $e$ interacciones entre los agentes responsables (por ej. temperatura, precipitación, vientos del oeste) de la variabilidad ambiental durante los últimos 18.000 años cal AP. Las áreas ecotonales del sudoeste patagónico han sufrido severos cambios ambientales desde la transición Pleistoceno-Holoceno. Numerosas investigaciones para el área han señalado cambios en la extensión de los glaciares, en la temperatura y en la precipitación, asociados a variaciones en la intensidad de los vientos del oeste (westerlies) y a cambios en el régimen de incendios (por ej. Björck et al. 2012; Glasser et al. 2011; Kilian \& Lamy et al. 2012; Lamy et al. 2010; Markgraf \& Huber 2010; Masiokas et al. 2009; Schäbitz et al. 2013; Sottile et al. 2012, Tonello et al. 2009; entre otros) donde se ilustran posibles escenarios sobre la variabilidad ambiental desde el Glacial tardío.

Gran parte de los estudios paleoambientales relacionados con gradientes ambientales de vegetación, temperatura y precipitación para el sur de Patagonia, fueron llevados a cabo a partir del análisis de secuencias extraídas de lagos y turberas (Bamonte \& Mancini 2011a; Heusser 1995; Huber \& Markgraf 2003; Huber et al. 2004; Mancini 2009; Markgraf \& Huber 2010; McCulloch \& Davis 2001; Möller et al. 2010; Moreno et al. 2009; Moy et al. 2009; Schäbitz 1991; Sottile et al. 2012; Villa-Martínez \& Moreno 2007; Wille \& Schäbitz 2009; entre otros). Estos estudios ofrecen información relacionada principalmente a la dinámica del bosque de Nothofagus en relación a la variabilidad de las precipitaciones asociadas a los vientos del oeste. En la zona extra-andina, los cambios de las comunidades vegetales desde fines del Pleistoceno, han sido reconstruidos a partir de registros polínicos de cuevas y aleros que presentan 
evidencias de ocupación humana (de Porras 2010; de Porras et al. 2009, 2011; Mancini 1998, 2002, 2008; Mancini \& Trivi de Mandri 1994; Mancini et al. 2002; Paez et al. 1999; Prieto et al. 1998; Trivi de Mandri et al. 1994). Estos registros son el resultado de interacciones ambientales, climáticas y culturales que deben ser tenidas en cuenta para realizar reconstrucciones de la vegetación del pasado e inferir condiciones ambientales (de Porras et al. 2011, Navarro et al. 2000, 2001). Otros registros paleoambientales localizados en la estepa pertenecen a grandes cuerpos de agua. Las dos grandes cuencas estudiadas son lago Cardiel (Ariztegui et al. 2008; Beres et al. 2008; Gilli et al. 2001; 2005; Markgraf et al. 2003; Stine \& Stine 1990) y laguna Potrok Aike (Haberzettl et al. 2005; Mayr et al. 2007, Schäbitz et al. 2013; Wille et al. 2007). Las inferencias basadas en la interpretación de las asociaciones polínicas fósiles en sitios arqueológicos han sido ampliamente cuestionadas por los procesos que las afectan (López Sáez et al. 2003). Sin embargo, las secuencias polínicas fósiles de cuevas y abrigos rocosos han sido usadas para reconstruir comunidades vegetales del pasado y condiciones ambientales en regiones áridas y semiáridas, considerando los procesos tafonómicos que actúan sobre ellas (de Porras 2010; Mancini et al. 2012). Además, la correlación entre los espectros polínicos de secuencias arqueológicas y de secuencias naturales o no arqueológicas (turberas, lagos, etc) permite identificar las alteraciones tafonómicas o antrópicas de los sitios arqueológicos (Mancini et al. 2012).

La influencia de los grupos-cazadores recolectores sobre las comunidades vegetales ha sido mencionada como factor secundario respecto a los factores ambientales (climáticos) para el sur de Patagonia (Huber \& Markgraf 2003). Las autoras sostienen que el incremento de la temperatura a comienzos del Holoceno pudo haber tenido un efecto significante favoreciendo la propagación de fuegos naturales, desligando al hombre como factor principal de los cambios en las comunidades vegetales. Markgraf \& Anderson (1994) interpretaron a las diferencias regionales en la ocurrencia de fuegos postglaciales como factores naturales en gradientes climáticos. Por el contrario, Heusser $(1987,1994)$ propuso al impacto humano generado por cazadoresrecolectores como factor primario necesario para la ocurrencia de los incendios posglaciales. Actualmente, la modificación de la vegetación natural en Patagonia debido a la intervención humana es evidente. La conquista española durante el siglo XIV dejó al descubierto grandes áreas para la colonización y asentamiento europeo, dando paso a una era de explotación (Borrelli \& Oliva 2001). Sin embargo, el mayor efecto antrópico sobre la vegetación por el asentamiento europeo ha sido principalmente observado en el siglo XX.

Este trabajo tiene como objetivo presentar la información paleoambiental obtenida a partir del análisis polínico de secuencias arqueológicas de dos sitios en reparos rocosos en el área del lago San Martín $\left(49^{\circ} \mathrm{S} ; 72^{\circ} \mathrm{O}\right.$, provincia de Santa Cruz, Argentina) e integrar esta información a un marco paleoambiental y a la información precedente sobre las ocupaciones humanas para la región de los lagos Tar y San Martín.

\section{CARACTERÍSTICAS DEL AMBIENTE}

La cuenca del lago San Martín (Fig. 1) está rodeada por distintos tipos de geoformas superficiales. Desde el hielo continental hasta el costado occidental del lago se encuentra un paisaje montañoso de alturas medias que oscilan entre los 1500 y 2500 msnm. Al bordear el lago por sus costas sur y este se observa un paisaje ondulado modificado por la acción glaciaria, rodeado por taludes y laderas rocosas con pendientes fuertes (Movia et al. 1987; Pereyra et al. 2002).

El clima del área se caracteriza por tener una temperatura media de $4^{\circ} \mathrm{C}$ y un marcado gradiente de precipitación que varía desde alrededor $800 \mathrm{~mm}$ anuales en el límite con Chile (lago O' Higgins), a menos de $200 \mathrm{~mm}$ en el lado oriental del lago Tar (Fig. 1). La cordillera de los Andes genera un importante efecto de sombra de lluvia sobre la Patagonia argentina. Por lo tanto, el lado occidental de los Andes presenta condiciones húmedas a hiper-húmedas de entre 5.000-10.000 mm de precipitación anual. Estas condiciones favorecen el establecimiento de bosques siempre verdes y alimentan a numerosos glaciares y a los campos de hielo Norte, Sur, y de la cordillera de Darwin. La precipitación anual desciende a menos de $300 \mathrm{~mm}$ a pocos kilómetros al este de la cordillera favoreciendo el reemplazo de la vegetación boscosa por estepas graminosas y arbustivas. Estas condiciones se extienden a través de la meseta central hasta la 
costa Atlántica (Garreaud et al. 2013).

Las unidades de vegetación están relacionadas con el gradiente de precipitación que disminuye hacia el este (Fig. 1). Al occidente del lago, se desarrolla el bosque de Nothofagus. El bosque que se encuentra en el oeste en los alrededores de Estancia La Maipú (Fig. 1), está caracterizado por Nothofagus pumilio y presenta una diversidad relativamente alta de otras especies. En el sector oriental del lago se observa el desarrollo del bosque en península Chacabuco (Fig. 1) que, en contacto con la estepa graminosa, presenta elementos característicos del ecotono bosque-estepa. En el área occidental de la margen norte del lago el bosque presenta las mayores densidades de árboles.

En los valles glaciares que bordean al lago por sus costas sur y este se desarrolla la estepa graminosa (Fig. 1) que está dominada por Festuca pallescens acompañada por Festuca argentina y el arbusto Nardophyllum obtusifolium. Otros pastos encontrados en el área son Poa ligularis, Stipa chrysophylla y Festuca pyrogea. También pueden hallarse Carex andina, Polygala darwiniana y Nassauvia darwinii y en algunos casos, Junellia tridens, Senecio filaginoides y Berberis heterophylla (Movia et al. 1987).

Entre los lagos San Martín y Tar, la estepa sub-arbustiva (Fig. 1) está caracterizada por Nassauvia glomerulosa asociada a Festuca pallescens, Stipa sp. y Poa sp. (Movia et al. 1987). Plantago patagonica y $P$. correae son especies nativas de esta unidad de vegetación (Correa 1999).

Las estepas arbustivas que se desarrollan en el área se encuentran al este de la graminosa. Estas estepas arbustivas, como la que se desarrolla en los alrededores de Estancia La Lila (Fig. 1) están asociadas principalmente a laderas rocosas donde predomina Nardophyllum obtusifolium asociado a F. pallescens con alta proporción de Berberis heterophylla, Senecio filaginoides y Mulinum spinosum que tapizan las laderas de altiplanicies y terrazas (Movia et al. 1987). También se observan Berberis buxifolia, Senecio sp., Adesmia boronioides, Acaena pinnatifida, Taraxacum sp., Poaceae (como Hordeum comosum, Stipa ibari, S. neaei, S. speciosa, Poa ligularis y Bromus setifolius; Movia et al. 1987), Plantago lanceolada y Cerastium arvense, entre otras.

Asociados a esta última unidad de vegetación se encuentran los sitios arqueológicos Bloque
1-Oquedad (Belardi et al. 2010) y Cueva del Paisano Desconocido (Espinosa et al. 2013). El primero se ubica en la margen sur del lago San Martín en el Campo de Bloques 1 (Belardi et al. 2010; Fig.1), es un bloque partido con reparo, orientado hacia el norte y una superficie de 10,5 m x 2,8 m (Belardi \& Campán 2009; Belardi et al. 2010). Comprende un paquete sedimentario de origen eólico relativamente homogéneo que descansa sobre una capa de till. La cronología del sitio fue presentada por Belardi et al. (2010) mediante dataciones radiocarbónicas sobre huesos de guanaco (Lama guanicoe) que mostraban modificaciones antrópicas. La evidencia arqueológica analizada indica que en el sitio se realizaban actividades relacionadas con la regularización y mantenimiento de artefactos (Pallo 2008, 2009). El sitio se encuentra ubicado en el límite entre la estepa graminosa y una estepa arbustiva asociada a laderas y valles glaciares. La primera está caracterizada por Festuca pallescens acompañada por elementos leñosos dispersos, mientras que en la segunda predomina Nardophyllum obtusifolium asociado a F. pallescens. Rodeando al lago San Martín, esta estepa arbustiva presenta mayor proporción de arbustos como Berberis heterophylla, Senecio filaginoides y Mulinum spinosum que tapizan las laderas rocosas (Movia et al. 1987). El segundo sitio, Cueva del Paisano Desconocido se encuentra en el extremo noreste del lago, sobre la bahía La Lancha (Espinosa et al. 2013; Fig. 1). La cueva presenta una boca de $14 \mathrm{~m}$ y una profundidad de 16,9 m, alcanzando un máximo de 2,10 m en su parte más protegida. El sedimento es mayormente coluvial, eólico laminar con lente areno-arcillosa. Se realizaron fechados radiocarbónicos también sobre huesos de L. guanicoe con modificaciones antrópicas con el fin de establecer la cronología para el sitio. El registro arqueológico obtenido en esta secuencia también indica el uso logístico de este espacio aunque con menor intensidad. Estos sitios podrían haber funcionado como campamentos intermedios a los campos altos y al interior del bosque (Espinosa et al. 2013). La cueva se encuentra en una estepa arbustiva que presenta las mismas características que aquella que linda con el sitio Bloque 1-Oquedad. Desde la boca de la cueva que se orienta en dirección oeste, se puede observar el bosque que se desarrolla en las laderas de la península Chacabuco (Fig. 1). 


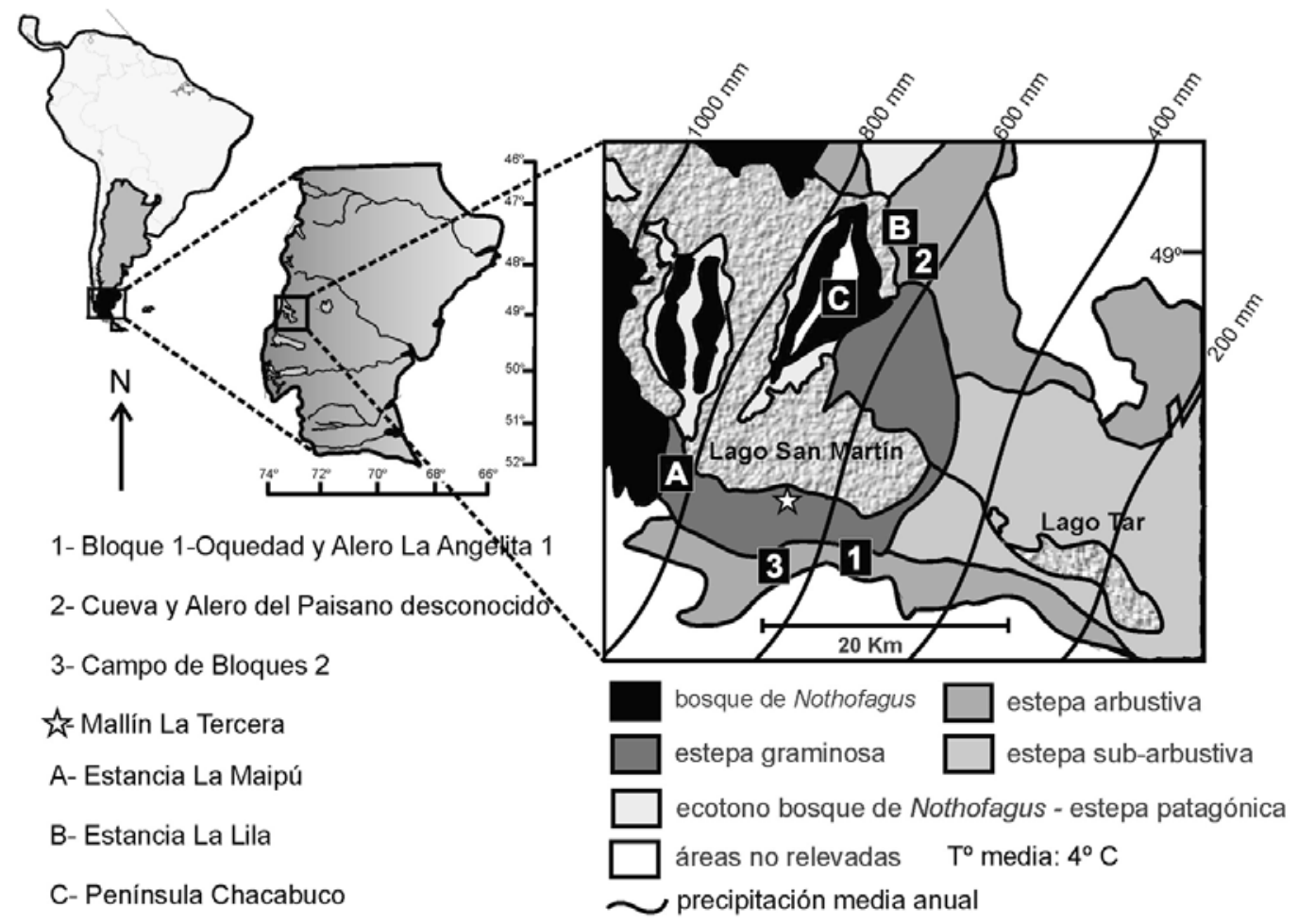

Fig. 1. Área del lago San Martín. En el mapa se observan las principales unidades de vegetación (modificado de Movia et al. 1987) asociadas al gradiente de precipitación que disminuye hacia el este. Con números (1-3) se ubican los sitios fósiles considerados en este trabajo y con letras $(\mathrm{A}-\mathrm{C})$ puntos nombrados en el texto.

INFORMACIÓN PALEOAMBIENTAL PARA EL ÁREA DEL LAGO SAN MARTÍN

Según lo inferido a partir de los análisis polínico y de partículas de carbón vegetal sedimentario (Fig. 2) realizados para el área a partir de la secuencia Mallín La Tercera (49 $11^{\circ}$ ' S; $72^{\circ} 22^{\prime}$ O; Bamonte \& Mancini 2011a; Sottile et al. 2012) se pudo establecer cómo fue variando la composición y sucesión de las comunidades vegetales del área de la cuenca del lago San Martín desde la transición Pleistoceno-Holoceno.

Para la transición Pleistoceno-Holoceno (11.300-10.000 años cal AP) se infirieron condiciones muy áridas por el desarrollo de una estepa subarbustiva con dominancia de Poaceae y sub-arbustos, principalmente Ephedra (Fig. 2). Este tipo de vegetación sugiere que el actual límite entre la estepa graminosa y la sub-arbustiva, que actualmente se ubica entre el extremo oriental del lago San Martín y el occidental del Tar, estuvo desplazado hacia el oeste. A comienzos del Holoceno (10.000-9.500 años cal AP) se registró el reemplazo de la estepa sub-arbustiva por una de arbustos medianos y/o altos (caracterizada polínicamente por Asteraceae subf. Asteroideae) sugiriendo un aumento de la temperatura (Fig. 2). Durante el Holoceno temprano (9.500-8.000 años cal AP) se desarrolló una estepa graminosa y comenzaron a establecerse las distintas unidades de vegetación: el bosque en la zona andina (de acuerdo al incremento de Nothofagus), la estepa graminosa y la sub-arbustiva hacia el este, sugiriendo un aumento en la disponibilidad de humedad. El incremento de Poaceae y de otras hierbas y la disminución de arbustos y sub-arbustos indican condiciones de mayor disponibilidad de humedad (Fig. 2). Durante el Holoceno medio (8.000-4.000 años cal AP) y comienzos del tardío (4.000-3.000 años cal AP) las reconstrucciones señalan el desarrollo de una estepa con arbustos (Asteraceae subf. Asteroideae) 
altamente representados (Fig. 2), relacionado con condiciones de menor disponibilidad de humedad en sectores extra-andinos, mientras que en la zona cordillerana, las condiciones habrían sido más húmedas permitiendo la expansión del bosque. Secuencias ubicadas hacia el sudoeste en la zona andina, indican el desarrollo de un bosque denso hacia ca. 7.500 años cal AP (Huber \& Markgraf 2003; Markgraf \& Huber 2010; Moreno et al. 2009; Villa-Martínez \& Moreno 2007; Wille \& Schäbitz 2009). Durante el Holoceno tardío, a partir de los 3.000 años cal $\mathrm{AP}$, se establece una estepa graminosa dominada por Poaceae (Fig. 2) asociada a un aumento en la disponibilidad de humedad. Durante el último siglo se observan condiciones diferentes debido a un aumento en algunos arbustos y disminución de los pastos, mientras que en la zona andina, los menores valores de Nothofagus indican que el bosque podría haber sido más abierto, o bien haber tenido menor producción polínica. Esta señal de bosque abierto es registrada en Cerro Frías ubicado en el actual ecotono bosque-estepa (Mancini 2009), mientras que en sitios ubicados más hacia el sudoeste, el bosque comienza a declinar a partir de ca. 1.000 años cal AP (Huber
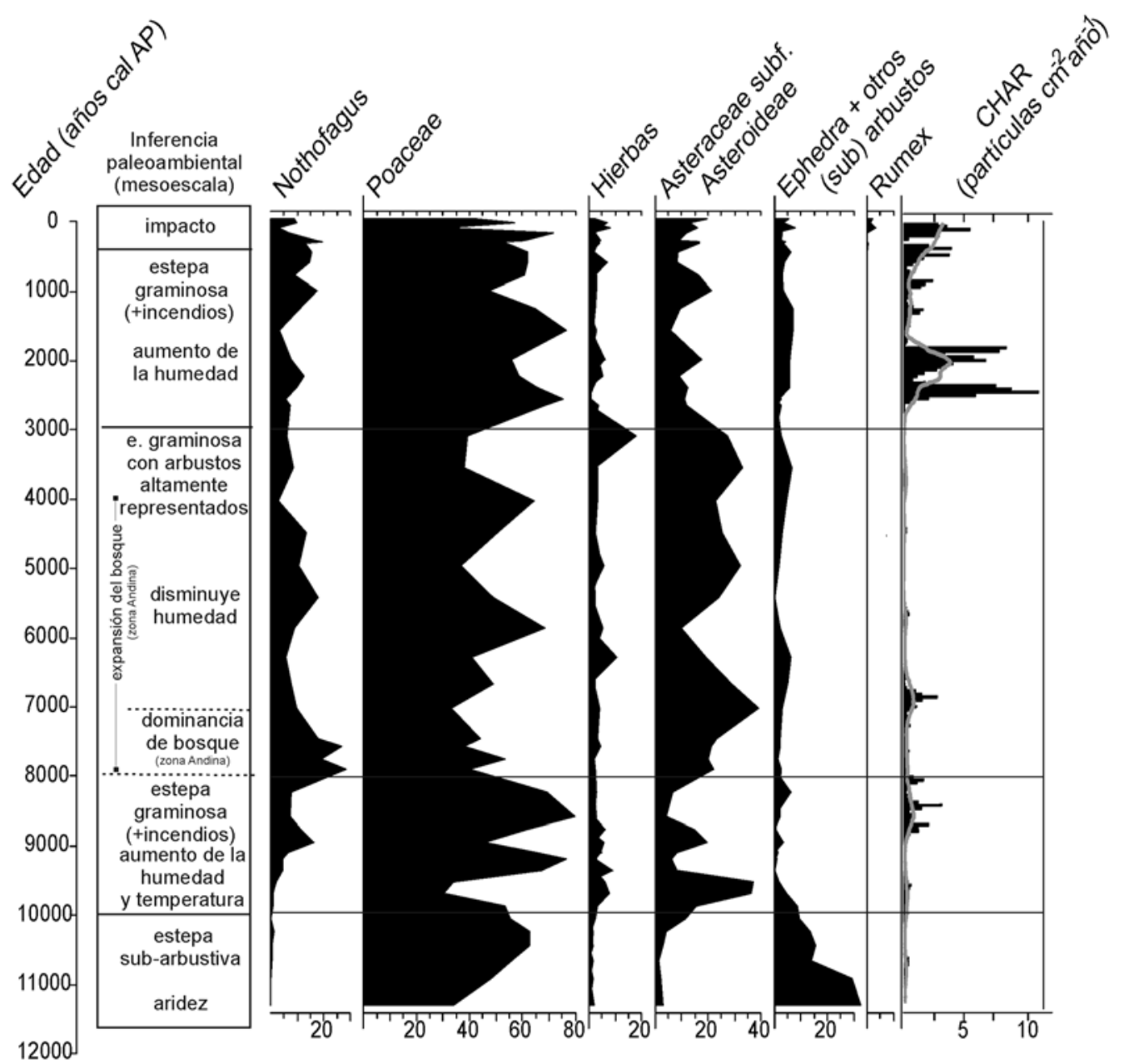

Fig. 2. Información paleoambiental inferida a partir del análisis polínico (Bamonte \& Mancini 2011a) y de partículas de carbón (Sottile et al. 2012) de Mallín La Tercera. Se presentan las principales variables polínicas (modificado de Bamonte \& Mancini 2011a) y la curva de carbón vegetal sedimentario (CHAR; modificado de Sottile et al. 2012) en función de la edad. 
et al. 2004; Villa-Martínez \& Moreno 2007; Wille \& Schäbitz 2009). La presencia de Rumex señala impacto antrópico, relacionado con el asentamiento europeo en la región (Fig. 2).

El análisis de partículas de carbón vegetal sedimentario para la secuencia Mallin La Tercera (Sottile et al. 2012) permitió evaluar la relación entre la tasa de acumulación (CHAR) y la continuidad de combustible. En aquellos períodos donde se desarrolló una estepa graminosa (Holoceno temprano y tardío), los valores de CHAR son más altos, lo que refleja la ocurrencia de fuegos superficiales (Fig. 2). Estos fuegos pudieron haberse expandido por la continuidad que proporcionan las gramíneas a modo de combustible en un paisaje en el cual eran dominantes. Mientras que en los momentos de mayor desarrollo de arbustos como durante la transición Pleistoceno-Holoceno y Holoceno medio, el fuego se vio impedido por la configuración de una vegetación en parches (Fig. 2). Independientemente del origen, el fuego está controlado por las condiciones del combustible que afectan su propagación. Estudios realizados por Clark et al. (2002) señalan que durante períodos secos, la productividad de los pastos declina, la erosión aumenta y la limitante en el combustible reduce la importancia de los fuegos.

Las interpretaciones generadas a partir de los análisis realizados por Bamonte \& Mancini (2011a) y Sottille et al. (2012) presentan una estrecha relación con la variabilidad en la intensidad de los vientos del oeste. Para el Holoceno medio, la mayor intensidad de estos vientos (Moy et al. 2009) produjo un gradiente de precipitación bien marcado descargando las precipitaciones en el área cordillerana y generando condiciones más secas hacia el este, dando lugar al desarrollo del bosque en la cordillera y una estepa con arbustos en el área del lago San Martín. Mientras que, durante el Holoceno temprano y tardío, los vientos fueron menos intensos (Moy et al. 2009), por lo que las precipitaciones habrían alcanzado zonas más orientales, generando un gradiente de precipitación más suave, que influyó en las unidades de vegetación; el bosque disminuyó en la zona andina, mientras que en la zona extra-andina de la cuenca del lago, el aumento de la humedad efectiva favoreció el desarrollo de una estepa graminosa. Por otra parte, los cambios en la temperatura asociados a los eventos neoglaciares del Holoceno tardío (Aniya 1995; Glasser et al. 2004; Masiokas et al. 2009;
Porter 2000) podrían haber influido en la densidad del bosque y/o en la productividad polínica del mismo.

\section{MATERIALES Y MÉTODOS}

Se analizó el contenido polínico de 15 muestras de una secuencia del sitio Bloque 1-Oquedad y de 10 muestras de la Cueva del Paisano Desconocido. La toma de muestras fue realizada cada $5 \mathrm{~cm}$ en perfiles provenientes de excavaciones de $1 \mathrm{~m}^{2}$. En el caso del Bloque 1-Oquedad, la excavación fue realizada en la parte media del alero y contra la pared del mismo, mientras que en la Cueva del Paisano Desconocido la excavación se realizó sobre el lateral derecho cercano a la entrada de la cueva.

Para el análisis de las muestras se pesaron entre 15 y 20 gramos de cada una. Se les agregaron tres pastillas de Lycopodium clavatum, con el fin de calcular la concentración polínica por gramo de sedimento (Stockmarr 1971). La extracción polínica se realizó siguiendo el procedimiento descrito por Faegri \& Iversen (1989) que consiste en: filtrar las submuestras por malla de $120 \mu$ con agua destilada, deflocular las arcillas y eliminar los ácidos húmicos con $\mathrm{KOH} 10 \%$, eliminar los carbonatos con $\mathrm{HCl} 10 \%$, separar la fracción mineral por flotación con $\mathrm{ZnCl}_{2}(\delta$ $=2 \mathrm{~g} / \mathrm{ml}$ ), eliminar los silicatos con HF y finalmente realizar acetólisis para eliminar la materia orgánica. Se realizó el conteo polínico bajo microscopio óptico. Las sumas polínicas variaron entre 230 y 340 granos, con excepción de las muestras 6 y 7 de la cueva que presentaron sumas de 107 y 102 granos respectivamente. Los diagramas polínicos en porcentaje y las curvas de concentración polínica total (granos/gramo de sedimento) fueron realizados con el programa TILIA y TILIAGRAPH (TGView 2.0.0, Grimm 2004). Cada taxón fue expresado como porcentaje de la suma polínica total. Podocarpus, no fue incluido en la suma polínica por representar a un taxón extra-regional. Rumex también fue excluido ya que está relacionado al impacto antrópico. En el caso particular de la Cueva del Paisano Desconocido se excluyeron a Blechnum y Urticaceae por encontrarse actualmente en la superficie de la cueva (locales).

Las cronologías de las secuencias (edades radiocarbónicas) fueron tomadas de Belardi et al. (2010) y Espinosa et al. (2013). Las edades radiocarbónicas fueron calibradas usando el software CALIB 5.0.2 (Stuiver \& Reimer 1993; Stuiver et al. 
2005) mediante la curva del Hemisferio Sur (SHCal04; Mc Cormac et al. 2004). En el caso particular de la datación más antigua del sitio Bloque 1-Oquedad se utilizó la curva del Hemisferio Norte (IntCal04; Reimer et al. 2004).

\section{RESULTADOS}

\section{Bloque 1-Oquedad}

La zona inferior (Muestras 15 a 6) presenta los mayores valores de Asteraceae subf. Asteroideae
(25 a 90 \%) asociada a Poaceae (10 a $20 \%$ ) y subarbustos que actualmente son poco comunes en el área como Nassauvia, Ephedra y Chenopodiaceae, los cuales presentan valores que alcanzan el $5 \%$ en algunas muestras. Mulinum varía del 5 al $10 \%$. Cyperaceae, Caryophyllaceae y Rubiaceae, alcanzan valores cercanos al $10 \%$ en la base de la zona y luego disminuyen a menos de $5 \%$. El resto de las hierbas como Acaena, Lamiaceae, Asteraceae subf. Cichorioideae, Ranunculaceae, Apiaceae y Monocotyledoneae presentan valores cercanos al $5 \%$ en algunas de las muestras. La concentración
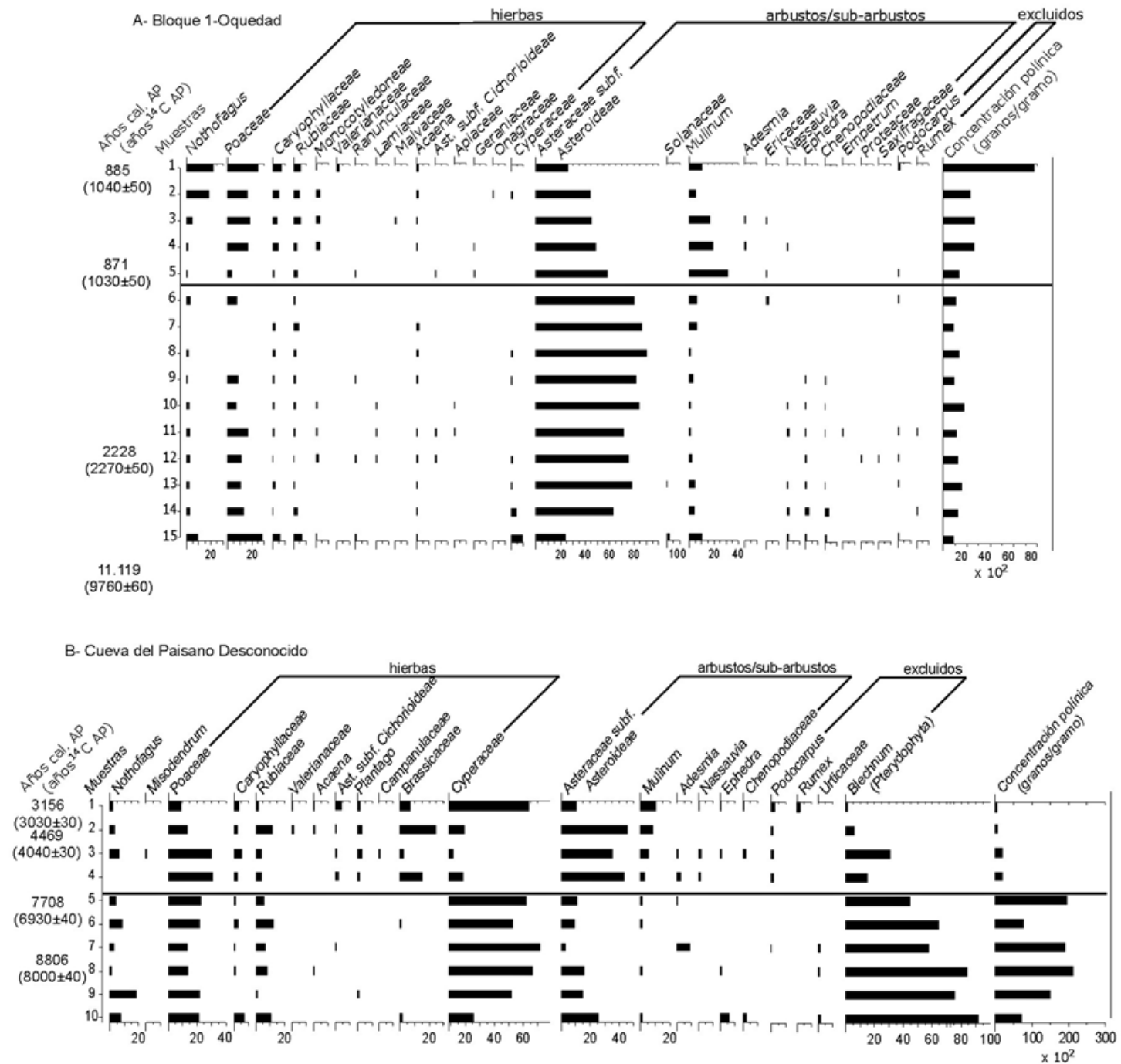

Fig. 3. Diagramas polínicos en porcentajes y curva de concentración polínica total de las secuencias arqueológicas: A- Bloque 1-Oquedad y B- Cueva del Paisano Desconocido, en función de las edades calibradas (años cal AP) y su correspondiente fechado rediocarbónico. 
polínica total se mantiene entre el 10 y $20 \times 10^{2}$ g/gr (Fig. 3A).

La zona superior (Muestras 5 a 1) presenta una disminución de Asteraceae subf. Asteroideae (30-60\%) desde la base al tope y un incremento de Poaceae (5 a $30 \%$ ). Mulinum (5 a 35\%) incrementa sus valores respecto a la zona inferior. Hierbas como Caryophyllaceae, Rubiaceae y Monocotyledoneae alcanzan el $10 \%$. El resto de las hierbas presenta valores alrededor del $5 \%$. La concentración polínica total aumenta de 15 a $80 \times 10^{2} \mathrm{~g} / \mathrm{gr}$ en el tope de la zona (Fig. 3A).

\section{Cueva del Paisano Desconocido}

La zona inferior de la secuencia polínica de la cueva (Muestras 10 a 5), presenta valores entre 5 y 25\% de Asteraceae subf. Asteroideae y baja representación de arbustos y sub-arbustos con excepción de Adesmia que presenta el $10 \%$ en una muestra. Entre las hierbas, Cyperaceae varía entre $<20 \%$ y $65 \%$ y Poaceae entre el 15 y $<25 \%$. Caryophyllaceae y Rubiaceae alcanzan valores alrededor del 10\%; el resto de las hierbas presenta valores inferiores al 5\%. Nothofagus varía entre $<5 \%$ y $20 \%$. Blechnum (Pteridophyta encontrado en la superficie de la cueva) presenta valores entre el 45 y 95\%. La concentración polínica total presenta valores entre 75 y 225 x $10^{2} \mathrm{~g} / \mathrm{gr}$ (Fig. 3B).

La zona superior (Muestras 4 a 1) presenta valores de Asteraceae subf. Asteroideae entre el 10 y $45 \%$ y Mulinum alcanza el $10 \%$, mientras que el resto de los arbustos y sub-arbustos presentan valores menores al 5\%. Poaceae varía entre el 10 y $30 \%$ y Cyperaceae entre el 5 y el $45 \%$. Brassicaceae alcanza el 25\% mientras que Caryophyllaceae y Rubiaceae varían entre el 5 y $10 \%$. El resto de las hierbas varía entre el 5 y $10 \%$. Nothofagus presenta valores menores al $10 \%$. Blechnum alcanza el $30 \%$. La concentración polínica total disminuyó $(<20 \mathrm{x}$ $10^{2} \mathrm{~g} / \mathrm{gr}$ ) respecto a la zona anterior (Fig. 3B).

\section{DISCUSIÓN}

Interpretación paleoecológica de los registros polinicos

El registro arqueológico Bloque 1-Oquedad, ubicado en cercanías del Mallín La Tercera, presenta cambios de la vegetación local asociada a taludes y laderas rocosas. Estos ambientes rocosos ofrecen situaciones de reparo a la vegetación, generando condiciones particulares para el desarrollo de ciertas comunidades vegetales diferentes de las representantes a mesoescala y a escala regional. Sin embargo, las comunidades locales también son afectadas por variaciones climáticas que provocan cambios en la disponibilidad de humedad. En la secuencia polínica del sitio Bloque 1-Oquedad se observa un cambio a ca. 870 años cal AP. Con anterioridad a este momento, altos valores de Asteraceae subf. Asteroideae (probablemente Senecio filaginoides y/o Nardophyllum obtusifolium, característicos de este tipo de ambientes) y presencia de sub-arbustos (Nassauvia y Ephedra) indican condiciones de menor humedad. Posteriormente, cambios en los conjuntos polínicos (por aumento de Poaceae, Caryophyllaceae, Rubiaceae, Monocotyledoneae y Mulinum) sugieren condiciones de mayor disponibilidad de humedad local.

A partir de la interpretación del espectro polínico de la Cueva del Paisano Desconocido se puede inferir una relación entre dicha secuencia y la de Mallín La Tercera, ya que ambas reflejan tendencias similares a mesoescala (Fig. 4). Se observan condiciones de mayor disponibilidad de humedad para el Holoceno temprano (zona inferior de la secuencia) debido a los mayores valores de Poaceae y de las hierbas acompañantes. Este aumento en la humedad también es reflejado por altos valores de Blechnum, helecho que crece actualmente en la superficie de la cueva. La zona superior de la secuencia representa parte del Holoceno medio donde se ven reflejadas condiciones de menor disponibilidad de humedad por un incremento de los arbustos al igual que lo registrado en Mallín La Tercera (Fig. 4).

La secuencia Bloque 1-Oquedad refleja entonces condiciones locales de la vegetación, principalmente aquella asociada a las laderas rocosas que la rodean. Este sitio está orientado hacia el norte, por lo que no recibe directamente la influencia de los vientos del oeste y por lo tanto el aporte de Nothofagus es muy bajo; sin embargo, la tendencia en los valores de este taxón es la misma que se observa en la secuencia Mallín La Tercera. Por el contrario, la boca de la Cueva del Paisano Desconocido se encuentra orientada hacia el oeste, por lo que 
entrampa directamente el polen transportado por los vientos del oeste, aunque los valores de Nothofagus también son muy bajos. Así, la orientación de estos abrigos rocosos no parece ser un factor determinante en la depositación polínica en el interior de los mismos, tal como fue encontrado también en cuevas de la meseta central de Santa Cruz (de Porras et al. 2011). Probablemente, la composición polínica de los sedimentos de los sitios analizados esté influenciada principalmente por las características topográficas (altura, barreras) y la distribución de la vegetación del área, mientras que la concentración polínica de los mismos, lo esté por las condiciones de humedad y/o la tasa de depositación de los sedimentos.

Las secuencias arqueológicas consideradas en este trabajo representan ventanas temporales, por lo que la comparación con una secuencia no arqueológica (Mallín La Tercera) permite interpretar las variaciones observadas a partir del análisis polínico de los sitios arqueológicos y las escalas que representan (Fig. 4). La depositación continua de los sedimentos de secuencias de lagos y turberas/mallines permite la reconstrucción de la historia de la vegetación en el pasado con mayor resolución cronológica que las secuencias de sitios arqueológicos. En las secuencias arqueológicas consideradas en este estudio no se han registrado distorsiones significativas en los conjuntos polínicos por efecto antrópico. Los tipos polinícos hallados forman parte de la vegetación local y regional y la diversidad de los mismos es un carácter indicador de una buena preservación, probablemente debido a las características ambientales secas de la región.

\section{Paleoambientes y ocupaciones humanas}

La correspondencia de ocupaciones humanas para $\approx 11.100$ años cal AP (ca. 9.500 años ${ }^{14} \mathrm{C} \mathrm{AP}$ ) tanto para el área sur del Lago San Martín (Bloque 1-Oquedad; Belardi et al. 2010) como para otras áreas occidentales del Parque Nacional Perito Moreno (Cerro Casa de Piedra 7; Civalero \& Aschero 2003) y del Lago Argentino (Chorrillo Malo 2; Civalero \& Franco 2003; Franco \& Borrero 2003), demuestran la disponibilidad y exploración de estos espacios para este momento (Borrero 1989-1990). Para el área del lago San Martín, según lo inferido a partir de Mallín La Tercera, en el período dominó una estepa sub-arbustiva bajo condiciones áridas. Según la integración de lo inferido a partir de Mallín La
Tercera y Cueva del Paisano Desconocido, a fines del Holoceno temprano y principios del medio, se registró la expansión del bosque en la zona andina (Fig. 4) así como el incremento en la temperatura (Renssen et al. 2005). Estas condiciones podrían haber influido en las ocupaciones de los sitios más cercanos al bosque como es el caso de la Cueva del Paisano Desconocido (Fig. 4). Según Espinosa et al. (2013), dichos espacios habrían funcionado como última parada antes de ingresar al bosque y/o ascender a las mesetas. Esto genera un marco para plantear que el acceso al bosque de los grupos cazadoresrecolectores podría haberse dado en los momentos de expansión del mismo en busca de algún recurso particular. Existen otros estudios polínicos sobre secuencias sedimentarias analizadas para el área de la cuenca del lago San Martín. En la margen noreste del lago, Bamonte \& Mancini (2011b) estudiaron una secuencia sedimentaria de otro mallín, denominado Mallín del Paisano Desconocido el cual presenta características similares al denominado Mallín La Tercera. Si bien las dos secuencias presentan una extensión temporal distinta (solapándose desde el Holoceno medio), reflejan las mismas variaciones en el espectro polínico y, por consiguiente, en las comunidades de vegetación reconstruidas para los períodos de tiempo compartidos (Bamonte \& Mancini 2011b). Dicha secuencia proveniente de un mallín lindante a los sitios Cueva y Alero del Paisano Desconocido, presenta altos valores de Nothofagus que disminuyen hacia finales del Holoceno medio (Bamonte 2012; Bamonte \& Mancini 2011b; 2012). Estos resultados fortalecen las interpretaciones generadas a partir del Mallín La Tercera, las que se resumen en este trabajo.

Las edades correspondientes a niveles con ocupación para los sitios de la margen sur y noreste del lago San Martín (margen sur: Bloque 1-Oquedad; Alero La Angelita 1 del Campo de Bloques 1 -Belardi et al. 2010-, Bloques 1 y 2 del Campo de Bloques 2 -Belardi \& Campán, 2009-, margen noreste: Cueva y Alero del Paisano Desconocido -Espinosa et al. 2013-) indican una mayor intensidad de ocupación desde el Holoceno medio y durante el tardío (ver Belardi et al. 2013) bajo condiciones relativamente más húmedas (Fig. 4). A partir de los 3.000 años cal AP, se registró un aumento de la estepa graminosa por incremento de la humedad en la zona extra-andina y una disminución de la señal 


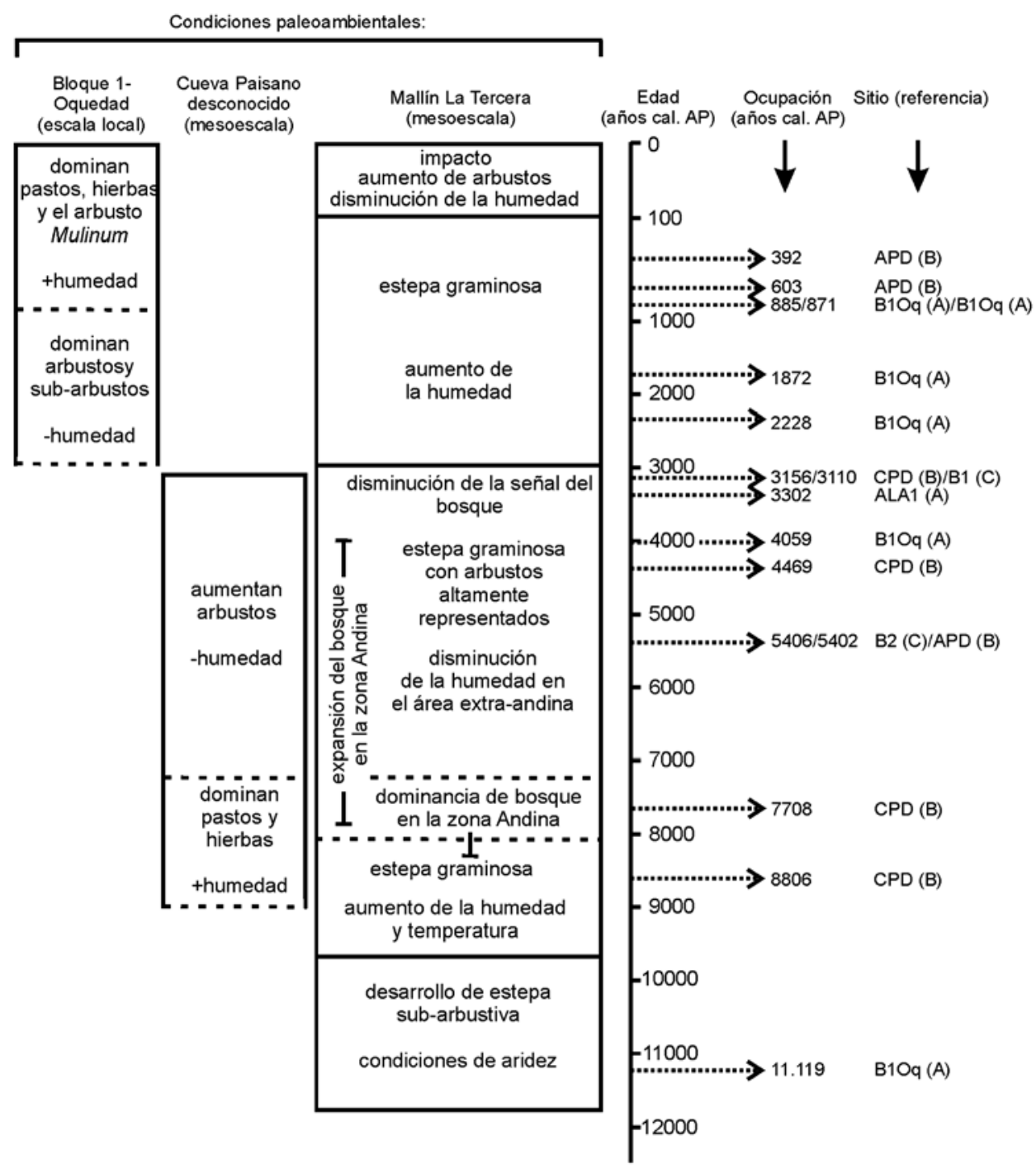

Fig. 4. Esquema donde se integran las condiciones paleoambientales inferidas a partir de Mallín La Tercera, Cueva del Paisano Desconocido y Bloque 1-Oquedad. Se acoplan los momentos de ocupación registrados para el área del lago San Martín a partir de los sitios: APD (Alero del Paisano Desconocido); B1Oq (Bloque 1-Oquedad); CPD (Cueva del Paisano Desconocido); B1 (Bloque 1); ALA1 (Alero La Angelita 1); B2 (Bloque 2). Las edades para estos sitios fueron tomadas de: A- Belardi et al. 2010, BEspinosa et al. 2013 y C- Belardi \& Campán 2009.

de bosque de la zona andina. No obstante, en los conjuntos polínicos de las secuencias analizadas no se observaron distorsiones significativas producto de las actividades de los grupos cazadores-recolectores.

\section{CONCLUSIONES}

A partir del análisis de la secuencia polínica del sitio Bloque 1-Oquedad fue posible reconstruir las variaciones de la vegetación de las laderas que lo circundan (escala local) para una parte del Holoceno tardío. En cambio, el análisis de la secuencia de la Cueva del Paisano Desconocido, brindó información a mesoescala similar a la obtenida de la secuencia Mallín La Tercera. El análisis polínico y de partículas de carbón vegetal sedimentario de este mallín (Bamonte \& Mancini 2011a; Bamonte 2012; Sottile et al. 2012), permitieron reconstruir las condiciones 
paleoambientales que reinaron en el área de estudio desde la transición Pleistoceno-Holoceno. Esta información pudo relacionarse con la obtenida a partir del análisis polínico de la cueva (mesoescala) y con los momentos de ocupación registrados para el área. De esta manera fue posible inferir variaciones en la vegetación y en el clima y evaluar la relación de estos cambios con la organización de las poblaciones. Si bien no se observan cambios en las comunidades de vegetación para los distintos momentos de ocupación de los grupos cazadores-recolectores, los principales signos de impacto antrópico se registran para el último siglo (a partir de Mallín La Tercera) y son coincidentes con el asentamiento europeo.

\section{AGRADECIMIENTOS}

A las familias Perazzo y Díaz (Ea. La Tercera), Vila Moret (Ea. La Angelita), Díaz (Eas. La Federica y La Lila) por la ayuda brindada durante los trabajos de campo. A Alejandro Súnico por su colaboración. A dos evaluadores anónimos por sus comentarios y sugerencias. Las investigaciones realizadas en este trabajo fueron llevadas a cabo con los siguientes subsidios: CONICET (PIP 1265, 6405 y 0418), Universidad Nacional de Mar del Plata (EXA-UNMdP 510/10), Universidad Nacional de la Patagonia Austral (UNPA-UARG 29/A245-1).

\section{BIBLIOGRAFÍA}

Aniya, M. (1995). Holocene glacial chronology in Patagonia: Tyndall and Uppsala glaciers. Artic and Alpine Research, 27 (4), 311-322.

Ariztegui, D., Anselmetti, F. S., Gilli, A. \& Waldmann, N. (2008). Late Pleistocene environmental change in eastern Patagonia and Tierra del Fuego - A limnogeological approach. En J. Rabassa (Ed.), The Late Cenozoic of Patagonia and Tierra del Fuego (pp. 241-253). Ámsterdam: Developments in Quaternary Science, Elsevier.

Bamonte, F. P. (2012). Cambios paleoecológicos y su posible relación con las ocupaciones humanas durante el Holoceno en el SO de Santa Cruz, Argentina. Tesis Doctoral, Facultad de Ciencias Exactas y Naturales, Universidad Nacional de Mar del Plata.

Bamonte, F. P. \& Mancini, M. V. (2011a). Palaeoenvironmental changes since Pleistocene-Holocene transition: pollen analysis from a wetland in Southwestern Patagonia (Argentina). Review of Paleobotany and Palynology,
165 (1-2), 103-110.

Bamonte, F. P. \& Mancini, M. V. (2011b). Historia paleoambiental en el área del lago San Martín (49 S, 72으) a partir de secuencias de mallines y sitios arqueológicos. VIII Jornadas de Arqueología de la Patagonia. Malargüe, Mendoza, Argentina.

Bamonte, F. P. \& Mancini, M. V. (2012). Cambios paleoambientales desde el Holoceno medio para el área del lago San Martín (49오 ${ }^{\circ}$; $2^{\circ}$ O): integración a una escala regional. XV Simposio Argentino de Paleobotánica y Palinología. Corrientes, Argentina.

Belardi, J. B. \& Campán, P. (2009). Resultados arqueológicos en el campo de Bloques 2, Sureste del Lago San Martín (Provincia de Santa Cruz). En M. Salemme, F. Santiago, M. Alvarez, E. Piana, M. Vazquez \& M. E. Mansur (Eds.), Arqueología de Patagonia: una mirada desde el último confín (pp. 927-939). Ushuaia: Editorial Utopías.

Belardi, J. B., Barrientos, G., Bamonte, F. P., Espinosa, S. \& Goñi, R. (2013). Paleoambientes y cronología de las ocupaciones cazadoras recolectoras de las cuencas de los lagos Tar y San Martín (Provincia de Santa Cruz). Intersecciones en Antropología. En prensa.

Belardi, J. B., Espinosa, S., Carvallo Marina, F., Barrientos, G., Goñi, R., Súnico, A., ...Campán, P. (2010). Las cuencas de los Lagos Tar y San Martín (Santa Cruz, Argentina) y la dinámica del poblamiento humano del sur de Patagonia: integración de los primeros resultados. Magallania, 38 (2), 137-159.

Beres, M., Gilli, A., Ariztegui, D. \& Anselmetti, F. S. (2008). The Lago Cardiel basin, Argentina ( $\left.49^{\circ} \mathrm{S}\right)$ : Origen and evolution revealed by high - resolution multichannel seismic reflection studies. Journal of South American Earth Sciences, 25, 74-85.

Borrelli, P. \& Oliva, G. (Eds.). (2001). Ganadería ovina sustentable en la Patagonia Austral. Tecnología de manejo intensivo (272 pp). Santa Cruz, Argentina: INTA.

Borrero, L. A. (1989-1990). Evolución cultural divergente en la Patagonia Austral. Anales del Instituto de la Patagonia, 19, 133-140.

Björck, S., Rundgren, M., Ljung, K., Unkel, I. \& Wallin, A. (2012). Multi-proxy analyses of a peat bog on Isla de los Estados, easternmost Tierra del Fuego: a unique record of the variable Southern Hemisphere Westerlies since the last deglaciation. Quaternary Science Reviews, 42, 1-14.

Civalero, M. T. \& Aschero, C. (2003). Early occupations at Cerro Casa de Piedra 7, Santa Cruz Province, Patagonia, Argentina. En L. Miotti, M. Salemme y N. Flegenheimer (Eds.), Where the South winds Blow. Ancient evidence of Paleo South Americans (pp. 141- 147). Texas: 
Center for the study of the First Americans, Texas A \& M University.

Civalero, M. T. \& Franco, N. V. (2003). Early human occupations in Western Santa cruz Province, Southernmost South America. Quaternary International, 109-110, 77-86.

Clark, J. S., Grimm, E. C., Donovan, J. J., Fritz, S. C., Engstrom, D. R. \& Almendinger, J. E. (2002). Drought cycles and landscape responses to past aridity on prairies of the northern Great Plains, USA. Ecology, 83, 595-601.

Correa, M. M. (Ed.). (1999). Flora Patagónica. Parte VI: Dicotiledóneas Gamopétalas (Ericaceae a Calyceraceae). Tomo VIII (536 pp). Buenos Aires: INTA.

De Porras, M. E. (2010). Dinámica de la vegetación de la Meseta Central de Santa Cruz durante los últimos 11.000 años: forzantes bióticos y abióticos. Tesis Doctoral, Facultad de Ciencias Exactas y Naturales, Universidad Nacional de Mar del Plata 137 pp.

De Porras, M. E., Mancini, M. V. \& Prieto, A. R. (2009). Vegetation changes and human occupation in the Patagonian steppe, Argentina, during the late Holocene. Vegetation History and Archaeobotany, 18(3), 235-244.

De Porras, M. E., Mancini, M. V. \& Prieto, A. R. (2011). Modern pollen analysis in caves at the Patagonian steppe, Argentina. Review of Palaeobotany and Palynology, 166, 335-343.

Espinosa, S. L., Belardi, J. B., Barrientos, G. \& Carballo, M. (2013). Poblamiento e intensidad de uso del espacio en la cuenca del Lago San Martín (Patagonia argentina): nuevos datos desde la margen norte. Comechingonia, $17(1), 105-121$.

Faegri, K. \& Iversen, J. (1989). Textbook of Pollen Analysis, 4th ed (314 pp). New York: John Willey and Sons.

Franco, N. V. \& Borrero, L. A. (2003). Chorrillo Malo 2: Initial peopling of the Upper Santa Cruz Basin, Argentina. En L. Miotti, M. Salemme \& N. Flegenheimer (Eds.), Where the South winds Blow. Ancient evidence of Paleo South Americans (pp. 149- 152). Texas: Center for the study of the first Americans, Texas A \& M University.

Garreaud, R. D., Lopez, P., Minvielles, M. \& Rojas, M. (2013). Large Scale Control on the âtagonia Climate. Journal of Climate, 26, 215-236.

Garreaud, R. D., Vuille, M., Compagnucci, R. \& Marengo, J. (2009). Present-day South American Climate. PALAEO 3 Special Issue (LOTRED South America), 281 (3-4), 180-195.

Gilli, A., Anselmetti, F. S., Ariztegui, D., Bradbury, J. P., Kelts, K. R., Markgraf, V. \& Mckenzie, J. A. (2001). Tracking abrupt climate change in the Southern Hemisphere: a seismic stratigraphic study of Lago Cardiel, Argentina
(49ㅇ). Terra Nova, 13, 443-448.

Gilli, A., Ariztegui, D., Anselmetti, F. S., Mckenzie, J. A., MarkgraF, V., Hajdas, I. \& Mc Culloch, R. (2005). MidHolocene strengthening of the Southern Westerlies in South America-Sedimentological evidences from Lago Cardiel, Argentina (49 ${ }^{\circ}$ S). Global and Planetary Change, 49, 75-93.

Glasser, N. F., Harrison, S., Winchester, V. \& Aniya, M. (2004). Late Pleistocene and Holocene palaeoclimate and glacier fluctuations in Patagonia. Global and Planetary Change, 43, 79-101.

Glasser, N. F., Jansson, K. N., Goodfellow, B. W., De Angelis, H., Rodnight, H. \& Rood, D. H. (2011). Cosmogenic nuclide exposure ages for marains in the Lago San Martín Valley, Argentina. Quaternary Research, 75, 636-646.

Grimm, E. (2004). Tilia y TGView 2.0.2. Software. Springfield: Illinois State Museum, Research and Collection Center.

Haberzettl, T., Fey, M., Lücke, A., Maidana, N., Mayr, C., Ohlendorf, C., ...Zolitschka, B. (2005). Climatically induced lake levelchanges during the last two millennia as reflect in sediments of Laguna Potrok Aike, southern Patagonia (Santa Cruz, Argentina). Journal of Paleolimnology, 33, 283-302.

Heusser, C. J. (1987). Quaternary vegetation of southern South America. Quaternary South America and Antartic Peninsula, 5, 197-221.

Heusser, C. J. (1994). Paleoindians and fire during the late Quaternary in southern South America. Revista Chilena de Historia Natural, 67, 435-443.

Heusser, C. J. (1995). Three Late Quaternary pollen diagrams from southern Patagonia and their paleoecological implications. Palaeogeography, Palaeoclimatology, Palaeoecology, 118, 1-24.

Huber, U. M. \& Markgraf, V. (2003). Holocene fire frequency and climate change at Río Rubens Bog, southern Patagonia. En T. T. Veblen, W. L. Baker, G. Montenegro, T. W. Swetnam (Eds.), Fire and climatic change in temperate ecosystems of the western Americas (pp. 357-380). New York: Springer Verlag.

Huber, U. M., Markgraf, V. \& Schäbitz, F. (2004). Geographical and temporal trends in Late Quaternary fire histories of Fuego-Patagonia. South America. Quaternary Science Reviews, 23, 191-198.

Kilian, R. \& Lamy, F. (2012). A review of Glacial and Holocene paleoclimate records from southernmost Patagonia (49-55은). Quaternary Science Reviews, 53, 1-23.

Lamy, F., Kilian, R., Arz, H. W., Francois, J - P., Kaiser, J. \& Prange, M. (2010). Holocene changes in the position and intensity of the southern westerly wind belt. Nature 
Geoscience, 3 (10), 695-699.

López Sáez, J. A., López García, P. \& Burjachs, F. (2003). Arqueopalinología: Síntesis crítica. Polen, 12, 5-35.

Mancini, M. V. (1998). Vegetational changes during Holocene in the Extra-Andean Patagonia, Santa Cruz Province, Argentina. Palaeogeography, Palaeoclimatology, Palaeoecology, 138(1-4), 207-219.

Mancini, M.V. (2002). Vegetation and climate during the Holocene in Southwest Patagonia, Argentina. Review of Paleobotany and Palynology, 122, 101-115.

Mancini, M. V. (2008). Cambios paleoambientales en el ecotono bosque-estepa: análisis polínico del sitio Cerro Casa de Piedra 7, Santa Cruz (Argentina). En F. Morello, M. Martinic, A. Prieto, y G. Bahamonde (Eds.), Arqueología de Fuego-Patagonia. Levantando piedras, desenterrando huesos... y develando arcanos (pp. 89-93). Punta Arenas: Ediciones CEQUA.

Mancini, M. V. (2009). Holocene vegetation and climate changes from a peat pollen record of the forest-steppe ecotone, Southwest of Patagonia (Argentina). Quaternary Science Reviews, 28, 1490-1497.

Mancini, M. V. \& Trivi De Mandri, M.E. (1994). Vegetación en el alero Río Pinturas: análisis polínico del Alero Cárdenas. En C. J. Gradin y A. M. Aguerre (Eds.), Contribución a la arqueología del río Pinturas. Provincia de Santa Cruz (pp. 48-62). Concepción del Uruguay: Búsqueda de Ayllu.

Mancini, M. V., Bamonte, F. P. \& Sottile, G. D. (2012). Paleoecología y ocupaciones humanas durante el Holoceno en el área cordillerana y Patagonia extra-andina entre los $49^{\circ} \mathrm{S}$ y $51^{\circ} \mathrm{S}$, Santa Cruz (Argentina). En L. A. Borrero y K. Borrazzo (Eds.), Bosques, montañas y cazadores: biogeografía en Patagonia Meridional (pp. 37-60). Buenos Aires: CONICET-IMHICIHU.

Mancini, M. V., Paez, M. M. \& Prieto, A. R. (2002). Cambios paleoambientales durante los últimos $7000{ }^{14} \mathrm{C}$ años en el ecotono bosque-estepa, $47^{\circ}-48^{\circ} \mathrm{S}$, Santa Cruz, Argentina. Ameghiniana, 39 (2), 151-162.

Markgraf, V. \& Anderson, L. (1994). Fire history of Patagonia: climate versus human cause. Revista do Instituto Geográfico do Sao Paulo, 15, 33-47.

Markgraf, V. \& Huber, U. M. (2010). Late and postglacial vegetation and fire history in Southern Patagonia and Tierra del Fuego. Palaeogeography, Palaeoclimatology, Palaeoecology, 297, 351-366.

Markgraf, V., Bradbury, P., Schwalb, A., Burns, S., Stern, C. H., Arizategui, D., ...Maidana, N. (2003). Holocene palaeoclimates of southern Patagonia: limnological and environental history of Lago Cardiel, Argentina. The
Holocene, 13, 581-591.

Masiokas, M. H., Luckman, B. H., Villalba, R., Delgado, S., Skavarca, P. \& Ripalta, A. (2009). Little Ice Age fluctuations of small glaciers in the Monte Fitz Roy and Lago del Desierto areas, south Patagonian Andes, Argentina, Palaeogeography, Palaeoclimatology, Palaeoecology, 281, 351-362.

Mayr, C., Wille, M., Haberzettl, T., Fey, M., Jansen, S., Lücke, A., ...Zolitschka, B. (2007). Holocene variability of the Southern Hemisphere westerlies in Argentinean Patagonia $\left(52^{\circ}\right.$ S). Quaternary Science Reviews, 26, 579-584.

McCormac, F. G., Hogg, A. G., Blackwell, P. G., Buck, C. E., Higham, T. F. G. \& Reimer, P. J. (2004). SHCal04 Southern Hemisphere Calibration 0 - 1000 cal BP. Radiocarbon, 46, 1087-1092.

McCulloch, R. D. \& Davies, S. (2001). Late-glacial and Holocene palaeoenvironmental change in the central Strait of Magallan, southern Patagonia. Palaeogeography, Palaeoclimatolology, Palaeoecology, 173, 143-173.

Möller, P., Hjort, C., Björck, S., Rabassa, J. \& Ponce, J. F. (2010). Late Quaternary glaciation history of Isla de los Estados, southeasternmost South America. Quaternary Research, 73, 521-534.

Moreno, P. I., François, J. P., Villa-Martínez, R. P. \& Moy, C. M. (2009). Millenial-sacale variability in Southern Hemisphere westerly wind activity over tha last 5000 years in SW Patagonia. Quaternary Science Reviews, 28, 25-38.

Movia, C., Soriano, A. \& León, R. (1987). La vegetación de la Cuenca del Río Santa Cruz (provincia de Santa Cruz, Argentina). Darwiniana, 28, 9-78.

Moy, C. M., Moreno, P. I., Dunbar, R. B., Kaplan, M. R., Francois, J-P., Villalba, R. \& Haberzettl, T. (2009). Climate Change in Southern South America during the Last Two Millennia. En F. Vimeux, F. Sylvestre \& M. Khodri (Eds.), Past Climate Variability in South America and Surrounding Regions. Developments in Palaeoenvironmental Research, 14, 353-393.

Navarro, C., Carrión, J. S., Navarro, J., Munuera, M. \& Prieto, A. R. (2000). An experimental approach to the palynology of cave deposits. Journal of Quaternary Science, 15 (6), 603-619.

Navarro, C., Munuera, M., Prieto, A. R. \& Carrión, J. S. (2001). Modern cave pollen in an arid environment and its application to describe palaeorecords. Complotum, 13, 7-18.

Paez, M. M, Prieto, A. R. \& Mancini, M. V. (1999). Fossil pollen from Los Toldos locality: a record of the Lateglacial transition in the Extra-Andean Patagonia. Quaternary Internacional, 53-54, 69-75.

Pallo, C. (2008). El conjunto lítico del sitio Bloque 1-Oquedad 
(lago San Martín, SO de la provincia de Santa CruzArgentina). Tesis de Licenciatura en Ciencias Antropológicas. Facultad de Filosofía y Letras, Universidad de Buenos Aires.

Pallo, C. (2009). La tecnología lítica del sitio Bloque 1-Oquedad (lago San Martín, Provincia de Santa Cruz). Resultados de la primera etapa de análisis. En M. Salemme, F. Santiago, M. Alvarez, E. Piana, M. Vazquez \& M. E. Mansur (Eds.), Arqueología de Patagonia: una mirada desde el último confín (pp. 369-381). Ushuaia: Editorial Utopías.

Paruelo, J. M., Beltrán, A., Jobbágy, E., Sala, O. \& Golluscio, R. (1998). The climate of Patagonia: general patterns and controls on biotic processes. Ecología Austral, 8, 85-101.

Pereyra, F. X., Fauqué, L. \& González Díaz, E. F. (2002). Geomorfología. En M. J. Haller (Ed.), Geología y Recursos Naturales de Santa Cruz. El Calafate: Sociedad Geológica Argentina.

Porter, S. C. (2000). Onset of Neoglaciation in the Southern Hemisphere. Journal of Quaternary Science, 15(4), 395-104.

Prieto, A. R., Stutz, S. \& Pastorino, S. (1998). Vegetación del Holoceno en la cueva Las Buitreras, Santa Cruz, Argentina. Revista Chilena de Historia Natural, 71, 277-290.

Reimer P. J., Baillie, M. G. L., Bard, E., Bayliss, A., Beck, J. W., Bertrand, C. J. H., ...Weyhenmeyer, C. E. (2004). IntCal04 terrestrial radiocarbon age calibration, 0-26 cal kyr BP. Radiocarbon, 46 (3), 1029-1058.

Renssen, H., Goosse, H., Fichefet, T., Masson- Delmotte, V. \& Koç, N. (2005). Holocene climate evolution in the high-latitude Southern Hemisphere simulated by a coupled atmosphere-sea ice-ocean-vegetation model. The Holocene, 15, 951-964.

Schäbitz, F. (1991). Holocene vegetation and climate in Southern Santa Cruz, Argentina. Bamberger Geographische Schriften, 11, 235-244.

Schäbitz, F. Wille, M., Francois, J - P., Habertzettl, T., Quintana, F., Mayr, C., ...Zolitschka. (2013). Reconstruction of palaeoprecipitation based on pollen transfer functions - the record of the last 16 ja from Laguna Potrok Aike, southern Patagonia. Quaternary Science Reviews, DOI: 10.1016/jquascirev.2012.12.006.
Sottile, G. D., Bamonte, F. P., Mancini, M. V. \& Bianchi, M. M. (2012). Insights into Holocene vegetation and climate changes at the Southeastern side of the Andes: Nothofagus Forest and Patagonian Steppe fire records. The Holocene special issue, 22 (11), 1309-1322.

Stine, S. \& Stine, M. (1990). A record from Lago Cardiel of climate change in southern South America. Nature, 345, 705-708.

Stockmarr, J., (1971). Tablets with spores used in absolute pollen analysis. Pollen Spores, 13, 615-621.

Stuiver, M. \& Reimer, P. J. (1993). Extended ${ }^{14} \mathrm{C}$ database and revised CALIB radiocarbon calibration program. Radiocarbon, 35, 215-230.

Stuiver, M., Reimer, P. J. \& Reimer, R. W. (2005). CALIB 5.0.2. http://calib.qub.ac.uk/calib/.

Tonello, M. S., Mancini, M. V. \& Seppä, H. (2009). Quantitative reconstruction of Holocene precipitation changes in southern Patagonia. Quaternary Science Reviews, 72, 410-420.

Trivi DE Mandri, M. E., Burry, L. S. \& Lombardo, M. C. (1994). Estudio palinológico preliminar del Alero Charcamata. En C. J. Gradin, \& A. M. Aguerre (Eds.), Contribución a la arqueología del río Pinturas. Provincia de Santa Cruz (pp. 193-201). Concepción del Uruguay: Búsqueda de Ayllu.

Villa-Martínez, R. P. \& Moreno, P. I. (2007). Pollen evidence for variatins in the southern margin of the westerly winds in SW Patagonia over the last 12,600 years. Quaternary Research, 68, 400-409.

Wille, M. \& Schäbitz, F. (2009). Late-glacial and Holocene climate dynamics at the steppe/forest ecotone in southernmost Patagonia, Argentina: the pollen record from a fen near brazo Sur, Lago Argentino. Vegetation History and Archaeobotany, 18, 225-234.

Wille, M., Maidana, N. I., Schäbitz, F., Fey, M., Haberzettl, T., Janssen, S., ...Zolitschka, B. (2007). Vegetation and climate dynamics in southern South America: The microfossil record of Laguna Potrok Aike, Santa Cruz, Argentina. Review of Paleobotany and Palynology, 146, 234-246. 
BAMONTE et al. 